Radial and Nonradial Pulsations as Probes of Stellar Physics

ASP Conference Series, Vol. 259, 2002

C. Aerts, T.R. Bedding, \& J. Christensen-Dalsgaard, eds.

\title{
A Study of Convection with $\delta$ Scuti Stars in Open Clusters: the Pleiades
}

\author{
J.-C. Suárez ${ }^{1}$, E. Michel ${ }^{1}$, G. Houdek ${ }^{2}$, Y. Lebreton ${ }^{1}$, F. Pérez \\ Hernández ${ }^{3}$ \\ ${ }^{1}$ Observatoire de Paris-Meudon, DASGAL, UMR 8633,F-92195 \\ Meudon, France \\ ${ }^{2}$ I.A., University of Cambridge, Cambridge, U.K. \\ ${ }^{3}$ Instituto de Astrofísica de Canarias, E-38200 La Laguna, Tenerife, \\ Spain
}

\begin{abstract}
In this work we propose a preliminary seismic investigation of $\delta$ Scuti stars in the Pleiades cluster, focusing on potential diagnostics of convection and core-overshooting. Taking into account the effect of fast rotation in the modelling, we compare observed frequencies for $4 \delta$ Scuti stars with radial linear instability predictions. A satisfying agreement is reached between the predicted ranges of unstable modes and those derived from observations for "low-mass" stars $\left(\sim 1.55 M_{\odot}\right)$. However, a strong disagreement is found for "high-mass" stars $\left(\sim 1.77 M_{\odot}\right)$, whatever the mixing length $(\alpha)$ value. These results are compared with previous ones obtained for Praesepe.
\end{abstract}

\section{Introduction}

Michel et al. (1999) compared observed modes for $\delta$ Scuti stars in the Praesepe cluster with unstable modes as obtained from a linear stability analysis including a nonlocal time-dependent treatment of convection. In the present paper, we extend this work to the Pleiades cluster, updating several aspects of the procedure. Following Soufi et al. (1998) we improve the rotation description in the oscillation computations. We take into account the effect of fast rotation in the photometric parameters as described in Pérez et al. (1999).

\section{Observations and modelling}

Observational data for the $\delta$ Scuti stars used in this work are summarized in Table 1. All data and references can be found in Fox et al. (2001 and these proceedings).

The evolution models for the Pleiades cluster have been computed (for a metallicity of $\mathrm{Z}=0.014$ ) using the evolution code CESAM (Morel, 1997). We used OPAL opacity tables (Iglesias \& Rogers, 1996) and the atmosphere is computed using the Eddington's $T(\tau)$ law. Models with $\left(\alpha, d_{o v}\right)=(1.61,0.2),(1.8,0.2)$ and $(1.61,0.1)$ will be called Standard (Std), $\alpha$ - and $d_{o v}$-models respectively. $d_{o v}$ and 
Table 1. Observational data. $N$ : number of frequencies found; $\nu$ : interval of observed frequencies in $\mu \mathrm{Hz} ; m_{V}$ : apparent magnitude (Johnson $\mathrm{V}$ filter); $v \sin i\left(\mathrm{~km} \mathrm{~s}^{-1}\right)$ : projected surface linear rotation velocity.

\begin{tabular}{ccccc}
\hline Star & $N$ & $\nu(\mu \mathrm{Hz})$ & $m_{V}$ & $v \sin i$ \\
\hline HD 23643 & 4 & {$[197.2,377.8]$} & 7.76 & 185 \\
HD 23607 & 7 & {$[216.3,444.1]$} & 8.26 & 6 \\
HD 23567 & 7 & {$[253.1,524.9]$} & 8.30 & 95 \\
HD 23156 & 7 & {$[242.9,529.1]$} & 7.51 & 187 \\
\hline
\end{tabular}

$\alpha$ are the overshooting and the mixing-length parameters respectively. An age of $130 \pm 30 \mathrm{Myr}$ is found to be representative of this cluster.

\subsection{The correction for the effect of rotation}

Since we work with fast rotators, a correction for the effect of rotation on the photomotric parameters is required (Michel et al., 1999). Following Pérez et al. (1999), we apply such a correction for the selected objects (Fig. 1). The correction gives us access to an estimation of masses, radii and rotation rates, parameters which are used to compute uniformly rotating models.

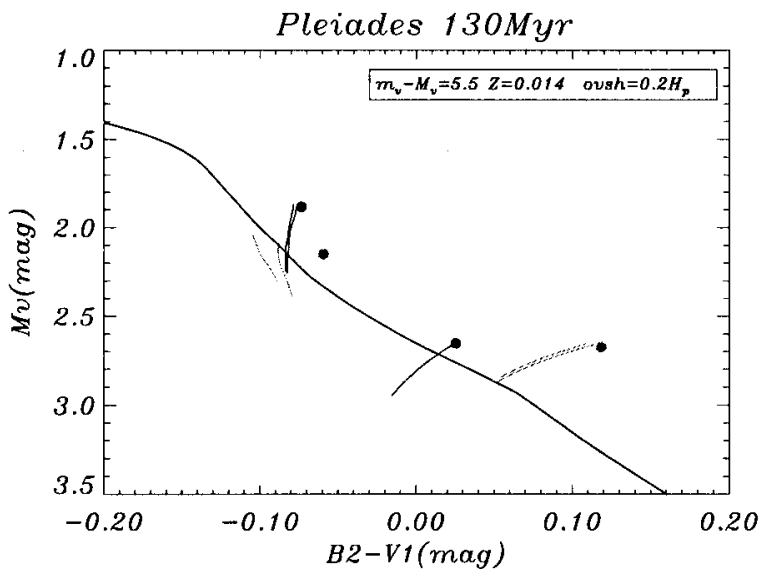

Figure 1. Corrections for the effect of rotation. Circles represent observed $\delta$ Scuti stars listed in Table 1 . Segments are potential position of their non-rotating counterparts. 


\subsection{The oscillations}

Rotating evolution models are used to compute adiabatic oscillation eigenfrequencies. Following Soufi et al. (1998), we improve the method used in Michel et al. (1999) to obtain these eigenfrequencies. The objective of such calculations is to determine a possible range of radial orders associated with the observed modes given in (Table 1 ).

On the other hand, in order to calculate linear growth rates, we use stability computations which are carried out in the manner described by Houdek et al. (1999). To do so, we compute envelope models fitted to our evolution models by outer convection zone dephts, masses, radii and effective temperatures.

\section{Results and analysis}

The fundamental parameters obtained in Sect.2.1 are not sensitive to realistic changes in age, $\alpha$ and $\boldsymbol{d}_{o v}$ parameters, as can be expected for such a young cluster. Thus, envelope models are computed for a unique set of parameters. The instability computation results will be sensitive to changes in $\alpha_{n l}{ }^{1}$ only through the fine description of the outer convective regions. They will not be affected by $d_{o v}$ variations, making useless the study of this parameter here. In this context, such clusters represent a good opportunity to test the convection in the outer convective zone, since they are free of uncertainties in the overshooting description.

Table 2. For $S t d$ and $\alpha$-models, $n_{E R M}$ : predicted radial orders from Evolutive Rotating Models; $n_{U A}$ : same from Unstability Computations ; $M$ : masses obtained from the correction for rotation.

\begin{tabular}{cccc}
\hline Star & $n_{E R M}$ & $n_{U A}$ & $M / M_{\odot}$ \\
\hline HD 23643 & {$[1,7-8]$} & {$[5,7-8]$} & $\sim 1.77$ \\
HD 23607 & {$[1,7-8]$} & {$[5,7-8]$} & $\sim 1.77$ \\
HD 23567 & {$[1,7-8]$} & {$[1,6-7]$} & $\sim 1.55$ \\
HD 23156 & {$[1,7-8]$} & {$[1,6-7]$} & $\sim 1.55$ \\
\hline
\end{tabular}

The predicted ranges of radial orders and those derived from observations are given in Table 2 for Std and $\alpha$-models. Contrary to the Praesepe results, a unique range of radial orders, including the fundamental, is obtained for the different masses. In the case of Praesepe, a good agreement between the predicted ranges and those derived from observations was found for the high-mass ${ }^{2}$ stars independently of the $\alpha_{n l}$ values. For low-mass stars, it was possible to reach an agreement for given $\alpha_{n l}$ values. Here, for the low-mass stars, a good

\footnotetext{
${ }^{1}$ The mixing length parameter associated with the non-local envelope model used in stability computations.

${ }^{2}$ In the case of Praesepe, where $\mathrm{Z}=0.019-0.03$, "high-mass" corresponds to $M>1.8 M_{\odot}$ and "low-mass" to $M \leq 1.8 M_{\odot}$.
} 
agreement is found, also independently of $\alpha_{n l}$, however, no matching is possible for high-mass stars and realistic $\alpha_{n l}$ values.

As in Michel et al. (1999), the previous discussion is limited to the results obtained for instability computations using fundamental parameters of non-rotating models. For the four $\delta$ Scuti stars of the Pleiades we have also computed rotating models, which present outer convective zones significantly deeper than the non-rotating models. Therefore, for these non-rotating models it is not possible to build consistent envelope models using the set of parameters described in section 2.2 without introducing, somehow, the effect of the centrifugal force.

\section{Conclusions and perspectives}

For selected $\delta$ Scuti stars in the Pleiades, we report here the results of a comparison between frequency ranges of unstable modes, predicted from instability computations, with observed ones. Significant differences are found with respect to results obtained for Praesepe (Michel et al., 1999). We find evidence suggesting that these results might be significantly changed when including some effects of rotation in the stability computations.

Taking advantage of the updated procedure presented in this paper, an homogeneous simultaneous study of the Pleiades and Praesepe is planned. This will give us the possibility of determine whether we can obtain consistent results and how they can constraint the description of the convective transport in the outer convective zones.

\section{References}

Michel, E., Hernández, M.M., Houdek, G., Goupil, M.J., Lebreton, Y., Pérez Hernández, F., Belmonte, J.A., \& Soufi, F. 1999, A\&A, 342, 153

Soufi, F., Goupil, M.J., \& Dziembowski, W.A. 1998, A\&A, 334, 911

Houdek, G., Balmforth, N.J., Christensen-Dalsgaard, J., Gough, D.O. 1999, A\&A, 351, 582

Iglesias, C.A. \& Rogers, F.J. 1996, ApJ464, 943

Fox Machado, L., Pérez Hernández, F., Suárez, J.-C., \& Michel, E. 2001, Proc. SOHO 10/GONG, (ESA SP-464), 427

Morel, P. 1997, A\&A, 124, 597

Pérez Hernández, F., Claret, A., Hernández, M.M., \& Michel, E. 1999, A\&A, 346,586

Suárez, J.-C., Michel, E. , Fox, L., Pérez, F., Lebreton, Y., Claret, A., \& Li, Z., 2001, Proc. SOHO 10/GONG, (ESA SP-464), 461 